\title{
Adverse anthropometric risk profile in biochemically controlled acromegalic patients: comparison with an age- and gender-matched primary care population
}

\author{
C. Dimopoulou • C. Sievers • H. U. Wittchen • \\ L. Pieper $\cdot$ J. Klotsche $\cdot$ J. Roemmler . \\ J. Schopohl · H. J. Schneider · G. K. Stalla
}

Published online: 4 February 2010

(C) The Author(s) 2010. This article is published with open access at Springerlink.com

\begin{abstract}
GH and IGF-1 play an important role in the regulation of metabolism and body composition. In patients with uncontrolled acromegaly, cardiovascular morbidity and mortality are increased but are supposed to be normalised after biochemical control is achieved. We aimed at comparing body composition and the cardiovascular risk profile in patients with controlled acromegaly and controls. A cross-sectional study. We evaluated anthropometric parameters (height, weight, body mass index (BMI), waist and hip circumference, waist to height ratio) and, additionally, cardiovascular risk biomarkers (fasting plasma glucose, HbA1c, triglycerides, total cholesterol, HDL, LDL, and lipoprotein (a), in 81 acromegalic patients (58\% cured) compared to 320 age- and gender-matched controls (ratio 1:4), sampled from the primary care patient cohort DETECT. The whole group of 81 acromegalic patients presented with significantly higher anthropometric parameters, such as weight, BMI, waist and hip circumference,
\end{abstract}

C. Dimopoulou and C. Sievers both authors contributed equally.

C. Dimopoulou - C. Sievers · H. J. Schneider - G. K. Stalla

Max Planck Institute of Psychiatry, Department of

Endocrinology, Munich, Germany

J. Roemmler · J. Schopohl · H. J. Schneider

Department of Endocrinology, Ludwig-Maximilians-University

of Munich, Munich, Germany

H. U. Wittchen · L. Pieper · J. Klotsche

Institute of Clinical Psychology and Psychotherapy, Technical

University Dresden, Dresden, Germany

C. Dimopoulou $(\square)$

Kraepelinstr. 2-10, 80804 Munich, Germany

e-mail: dimopoulou@mpipsykl.mpg.de but with more favourable cardiovascular risk biomarkers, such as fasting plasma glucose, total cholesterol, triglycerides and HDL levels, in comparison to their respective controls. Biochemically controlled acromegalic patients again showed significantly higher measurements of obesity, mainly visceral adiposity, than age- and gender-matched control patients (BMI $29.5 \pm 5.9$ vs. $27.3 \pm 5.8 \mathrm{~kg} /$ $\mathrm{m}^{2} ; \quad P=0.020$; waist circumference $100.9 \pm 16.8 v \mathrm{vs}$. $94.8 \pm 15.5 \mathrm{~cm} ; P=0.031$; hip circumference $110.7 \pm$ 9.9 vs. $105.0 \pm 11.7 \mathrm{~cm} ; P=0.001)$. No differences in the classical cardiovascular biomarkers were detected except for fasting plasma glucose and triglycerides. This effect could not be attributed to a higher prevalence of type 2 diabetes mellitus in the acromegalic patient group, since stratified analyses between the subgroup of patients with acromegaly and controls, both with type 2 diabetes mellitus, revealed that there were no significant differences in the anthropometric measurements. Biochemically cured acromegalic patients pertain an adverse anthropometric risk profile, mainly because of elevated adiposity measurements, such as BMI, waist and hip circumference, compared to an age- and gender-matched primary care population.

Keywords Acromegaly · GH · IGF-1 - Anthropometric parameters $\cdot$ Cardiovascular risk biomarkers

\section{Introduction}

Acromegaly, usually caused by a somatotroph pituitary adenoma, is an endocrine disorder with an estimated prevalence of 125 cases per million [1]. Due to the slowly progressive nature of the disease, it often takes 6.610.2 years until acromegaly is diagnosed [2]. Clinical 
presentation of acromegaly reflects chronic GH excess and comprises a broad spectrum of comorbidities, such as cardiovascular features (i.e. hypertension), metabolic features (i.e. diabetes mellitus), respiratory features (i.e. sleep apnea), bone and joint features, soft tissue and skin changes, malignancies, endocrine consequences (thyroid goiter) and a variety of neurological complications, such as nerve compression syndromes [3]. Unless adequate biochemical control is achieved, acromegaly is associated to elevated morbidity and mortality, mostly because of cardiovascular disease [4, 5].

Therapy for acromegaly represents a demanding task for the physician, since a subset of patients exhibit treatmentresistant disease. Management options include transsphenoidal surgery, representing the first-line treatment for acromegaly, radiation and medical therapy. Treatment with somatostatin analogues inhibits $\mathrm{GH}$ secretion and induces tumour shrinkage, whereas the $\mathrm{GH}$ receptor antagonist pegvisomant effectively blocks $\mathrm{GH}$ action in the periphery leading to disease control [6]. Effective control of GH and IGF-1 excess improves comorbidities as well as lowers mortality rates [7].

It is known that GH and IGF-1 play an important role in the regulation of metabolism and body composition [8]. $\mathrm{GH}$ exhibits lipolytic effects and can cause insulin resistance, whereas IGF-1 exhibits anti-lipolytic and insulinsensitising effects.

In acromegaly, an alteration of the body composition, including an increase in body water and lean body mass and a reduction in body fat, occurs.

Under therapy, and after the achievement of biochemical control, a reduction in body water and fat-free mass, and an increase in body fat have been reported [9, 10]. However, it is not clear if this increase results in higher adiposity measurements compared to a respective control group.

Few studies have examined anthropometric and cardiovascular risk parameters in patients with acromegaly under therapy. Recently, Freda et al. reported a lower visceral and subcutaneous fat mass in a cross-sectional study with 24 adults with active acromegaly compared to healthy controls. Sucunza et al. [8, 11] concluded from their cross-sectional study in 60 acromegalic patients that control of acromegaly reverts decreased fat mass. Regarding cardiovascular risk parameters, a more atherogenic lipoprotein profile in active acromegalic patients, consisting of higher levels of triglycerides and apolipoprotein B, was reported in the study of Boero et al. [12]. In another study, patients with active acromegaly presented with a higher insulin resistance index and fibrinogen levels than patients and subjects in control groups; CRP, leucocyte count, factor VIII and NT-pro BNP were similar in the three groups [13].
The aim of our study was to evaluate the cardiovascular risk profile of "biochemically cured" acromegalic patients by comparing their anthropometric parameters (height, weight, body mass index (BMI), waist and hip circumference, waist to height ratio), and cardiovascular risk biomarkers, to an age- and gender-matched primary care control group. Since acromegaly is associated with an increased risk of diabetes mellitus, which might influence the calculations, we performed stratified analyses in diabetic and non-diabetic patients separately.

\section{Subjects and methods}

\section{Participants}

Eighty-one patients with previously diagnosed acromegaly were recruited at the Endocrine Outpatient Unit of the Max Planck Institute of Psychiatry (MPIP) and the Department of Internal Medicine, Ludwig-Maximilians-University (LMU) in Munich. We sampled 320 age- and gender-matched control subjects in a fasting state (ratio 1:4) from the DETECT-cohort (Diabetes cardiovascular risk evaluation; targets and essential data for commitment and treatment, http://www.detect-studie.de/), a nationally representative cohort of patients attending a primary care practice in Germany [14, 15]. A 2 acromegalic patients had only two adequate DETECT-matches, so that the control group comprised of 320 instead of 324 subjects. The control groups for the subgroup analyses, as well as the diabetic control group, were recruited from the same DETECT-cohort $(n=320)$. All subjects gave their written informed consent. The study was approved by the local ethical committee.

Diabetes cardiovascular risk-evaluation: targets and essential data for commitment of treatment is a large, multistage, cross-sectional study of 55,518 unselected consecutive patients in 3,188 nationally representative primary care offices in Germany with a laboratory and follow-up component in a random subset of 7,519 patients. For all patients, a comprehensive standardised clinical evaluation (patients' self-report and physicians' assessments) was conducted. Patients in the prospective subset were additionally characterised by an extensive standardised laboratory programme. The main target of the DETECT study was the assessment of cardiovascular risk.

Assessment of comorbidities, biochemical variables and covariates

Clinical characteristics of the subjects were collected with regard to disease history, tumour characteristics, previous and current therapy, and comorbidities as well as current 
complaints. Comorbidities were diagnosed based on the physicians' assessments according to respective guidelines.

In the case of missing data or uncertainty, additional information was obtained by file review. Biochemical control in acromegalic patients was defined as (a) $\mathrm{GH}$ below $1 \mu \mathrm{g} / \mathrm{L}$ during a glucose tolerance test over $2 \mathrm{~h}$, or (b) age- and gender-adjusted normal values for IGF-1 within two standard deviations [16].

Regarding measurement of biochemical variables, serum concentrations of $\mathrm{GH}$ were measured using the automated Advantage chemiluminescent assay system (Nichols Diagnostics Institute, Bad Vilbel, Germany), and IGF-1 was determined on the continuous random access analyzer Immulite ${ }^{\circledR} 2000$ IGF-1 (Fa. DPC). Fasting plasma glucose and $\mathrm{HbA1c}$ were determined using the GLU and HBA1C II system of Roche, respectively. Cholesterol and triglycerides were also determined on a Roche analyser (CHOL, TG). Lipoproteins HDL and LDL were determined using the Roche HDL-C plus 3rd generation and LDL-C plus 2nd generation assay, respectively. Lipoprotein (a) was determined by nephelometry.

Within the DETECT-study, blood samples were collected and shipped by courier at room temperature within $24 \mathrm{~h}$ to the central laboratory (Medical University of Graz, Austria). Upon arrival, the samples were centrifuged immediately and serum and plasma were stored at $20^{\circ} \mathrm{C}$ until further processing. Clinical chemical parameters, as well as cholesterol and triglycerides, were determined on a Roche Modular automatic analyzer. Lipoproteins were determined electrophoretically on the HEENA SAS-3/ SAS-4 system. HbA1c was determined chromatographically on an ADAMS HA 8160 analysing system.

\section{Statistical analysis}

The statistical analysis was performed using the software package STATA 10.0 (Stata Corp). Statistical tests were conducted by logistic regression models for categorical variables and linear regression models for dimensional variables. Standard errors were estimated by Taylor linearization to account for the dependent clustered data structure.

\section{Results}

Demographic and clinical characteristics of the acromegalic study group

The acromegalic group $(n=81)$ comprised of 43 female (53.1\%) and 38 male patients (46.9\%) with a mean age of $54.7 \pm 1.4$ years. Patients with acromegaly had a high prevalence of pituitary macroadenomas (65\%). 73 patients $(90.1 \%)$ received transsphenoidal surgery as the primary therapy; the mean time after surgery was $10.15 \pm$ 0.88 years. 44 patients $(54.3 \%)$ received medical treatment including 10 patients $(12.3 \%)$ currently under dopamine agonists, 28 patients currently under somatostatin analogs and 9 patients $(11.1 \%)$ currently under pegvisomant. A 20 patients $(24.7 \%)$ were irradiated, 10 patients $(12.3 \%)$ received Gammaknife, 1 patient (1.2\%) Cyberknife and 5 patients $(6.2 \%)$ stereotactic fractionated radiatio. The mean time after radiotherapy was $9.1 \pm 1.61$ years. The acromegalic patients often suffered from arthralgia $(65.4 \%)$, pituitary deficiency $(59.3 \%)$, arterial hypertension $(54.3 \%)$, carpal tunnel syndrome $(45.7 \%)$, sleep apnea $(34.6 \%)$, diabetes mellitus $(27.2 \%)$ and malignancies $(11.1 \%)$.

Acromegaly was biochemically controlled in 49 patients $(58 \%)$ at the time of study. The mean actual GH and IGF-I SE scores were $2.8 \pm 0.59$ and $214.2 \pm 18.0 \mu \mathrm{g} / \mathrm{L}$, respectively.

Comparisons of anthropometric parameters and cardiovascular risk biomarkers between all acromegalic patients and primary care controls

Table 1 summarises the results on anthropometric parameters and laboratory findings. The whole group of 81 acromegalic patients presented with significantly higher anthropometric parameters, such as weight, BMI, waist and hip circumference, in comparison to their respective controls $\quad(86.6 \pm 17.8$ vs. $79.8 \pm 17.4 \mathrm{~kg} ; \quad P=0.002$; $29.2 \pm 5.2$ vs. $\quad 27.5 \pm 5.6 \mathrm{~kg} / \mathrm{m}^{2} ; \quad P=0.014 ; \quad 100.0 \pm$ 14.3 vs. $95.7 \pm 15.5 \mathrm{~cm} ; \quad P=0.026 ; 110.6 \pm 9.2$ vs. $105.6 \pm 12.0 \mathrm{~cm} ; P=0.001$, respectively).

Additionally, cardiovascular risk biomarkers, such as fasting plasma glucose, total cholesterol, triglycerides and HDL levels, were more favourable in the acromegalic group compared to the age- and gender-matched controls.

Regarding comorbidities, acromegalic patients suffered significantly more often from hypertension (54.3 vs. $37.8 \% ; P=0.008)$ and malignancies $(11.1$ vs. $3.1 \%$; $P=0.005)$, but less often from myocardial infarction (1.2 vs. $36.7 \% ; P=0.001)$, compared to their respective controls. In detail, the malignancies reported included two patients with skin cancer, one patient with colorectal carcinoma, one patient with renal carcinoma, one patient with thyroid carcinoma, one patient with cervical carcinoma, one patient with breast cancer, one patient with both breast and skin cancer and one patient with an unknown malignancy. 
Table 1 Comparisons of comorbidities, anthropometric parameters and cardiovascular risk biomarkers between patients with acromegaly $(n=81)$ and the age- and gender-matched DETECT-controls $(n=320)$

\begin{tabular}{|c|c|c|c|c|c|}
\hline & \multicolumn{2}{|c|}{ Patients with acromegaly $(n=81)$} & \multicolumn{2}{|c|}{ DETECT-control group $(n=320)$} & \multirow[t]{2}{*}{$P$-value } \\
\hline & $n$ & $\%$ & $n$ & $\%$ & \\
\hline \multicolumn{6}{|l|}{ Comorbidities } \\
\hline Hypertension & 44 & 54.3 & 121 & 37.8 & 0.008 \\
\hline Diabetes mellitus & 22 & 27.2 & 58 & 18.1 & 0.073 \\
\hline Coronary heart disease & 7 & 8.6 & 30 & 9.4 & 0.840 \\
\hline Myocardial infarction & 1 & 1.2 & 11 & 36.7 & 0.001 \\
\hline Cerebral insult & 4 & 4.9 & 0 & 0.0 & - \\
\hline Malignancies & 9 & 11.1 & 10 & 3.1 & 0.005 \\
\hline Pituitary deficiency & 48 & 59.3 & & & \\
\hline Corticotrope deficiency & 35 & 43.2 & & & \\
\hline Thyreotrope deficiency & 22 & 27.2 & & & \\
\hline Gonadotrope deficiency & 33 & 40.7 & & & \\
\hline \multirow[t]{2}{*}{ Growth hormone deficiency } & 3 & 3.7 & & & \\
\hline & & Mean (SD) & \multicolumn{2}{|r|}{ Mean (SD) } & $P$-value \\
\hline \multicolumn{6}{|l|}{ Anthropometric parameters } \\
\hline \multicolumn{2}{|l|}{ Height $(\mathrm{cm})$} & $172.4(12.0)$ & \multicolumn{2}{|r|}{$170.1(9.3)$} & 0.107 \\
\hline Weight (kg) & & $86.6(17.8)$ & \multicolumn{2}{|r|}{$79.8(17.4)$} & 0.002 \\
\hline $\mathrm{BMI}\left(\mathrm{kg} / \mathrm{m}^{2}\right)$ & & $29.2(5.2)$ & \multicolumn{2}{|r|}{$27.5(5.6)$} & 0.014 \\
\hline Waist circumference $(\mathrm{cm})$ & & $100.0(14.3)$ & \multicolumn{2}{|r|}{$95.7(15.5)$} & 0.026 \\
\hline Hip circumference $(\mathrm{cm})$ & & $110.6(9.2)$ & \multicolumn{2}{|r|}{$105.6(12.0)$} & 0.001 \\
\hline Waist to height ratio & & $0.58(0.09)$ & \multicolumn{2}{|r|}{$0.56(0.09)$} & 0.174 \\
\hline \multicolumn{6}{|l|}{ Biochemical parameters } \\
\hline IGF-1 $(\mu \mathrm{g} / \mathrm{L})$ & & $214.2(161.2)$ & & $131.5(54.3)$ & 0.000 \\
\hline \multicolumn{6}{|l|}{ Cardiovascular risk biomarkers } \\
\hline Fasting plasma glucose (mg/dL) & & $93.5(16.5)$ & & $104.1(35.1)$ & 0.001 \\
\hline HbA1c $(\%)$ & & $5.7(0.5)$ & & $5.6(1.0)$ & 0.550 \\
\hline Total cholesterol (mg/dL) & & $215.8(39.5)$ & & $226.6(44.0)$ & 0.045 \\
\hline Triglycerides (mg/dL) & & $121.1(63.6)$ & & $160.8(155.3)$ & 0.001 \\
\hline HDL (mg/dL) & & $60.4(21.4)$ & & $53.0(17.6)$ & 0.009 \\
\hline $\mathrm{LDL}(\mathrm{mg} / \mathrm{dL})$ & & $139.4(37.4)$ & & $131.0(32.4)$ & 0.093 \\
\hline Lipoprotein (a) (mg/dL) & & $36.1(43.5)$ & & $33.3(47.3)$ & 0.707 \\
\hline
\end{tabular}

Note: Significant effects are bold typed

Comparisons of anthropometric parameters and cardiovascular risk biomarkers between biochemically cured and uncontrolled patients and respective non-acromegalic controls

We went onto stratify the study group into controlled and uncontrolled patients and compared them to their respective primary care control patients.

When evaluating biochemically controlled acromegalic patients in comparison to their respective DETECT controls, anthropometric measurements such as weight (85.6 \pm 17.9 vs. $78.5 \pm 18.5 \mathrm{~kg} ; P=0.015)$, BMI $(29.5 \pm 5.9$ vs. $\left.27.3 \pm 5.8 \mathrm{~kg} / \mathrm{m}^{2} ; P=0.020\right)$, waist $(100.9 \pm 16.8$ vs. $94.8 \pm 15.5 \mathrm{~cm} ; P=0.031)$ and hip circumference
$(110.7 \pm 9.9$ vs. $\quad 105.0 \pm 11.7 \mathrm{~cm} ; \quad P=0.001) \quad$ were significantly higher in biochemically controlled patients. Cardiovascular risk biomarkers, such as fasting plasma glucose $(90.9 \pm 16.7$ vs. $104.8 \pm 39.3 \mathrm{mg} / \mathrm{dL} ; P=0.000)$ and triglycerides $(118.5 \pm 59.4$ vs. $153.8 \pm 123.1 \mathrm{mg} / \mathrm{dL}$; $P=0.006$ ), were significantly lower in biochemically controlled patients. Since radiation might also play a role in causing pituitary deficiency with effects on both body composition and cardiovascular risk biomarkers, we also performed the same analyses excluding patients who had been irradiated or were pituitary-deficient. We observed similar results with significant differences in BMI, waist and hip circumference between the groups $(P=0.039,0.015$ and 0.001 , respectively). 
Table 2 Comparisons of comorbidities, anthropometric parameters and cardiovascular risk biomarkers between biochemically controlled $(n=49)$ and uncontrolled patients $(n=31)$ and the age- and gender-matched DETECT-controls $(n=196$ and $n=120$, respectively)

\begin{tabular}{|c|c|c|c|c|c|c|c|c|c|c|c|c|}
\hline & \multicolumn{2}{|c|}{$\begin{array}{l}\text { Patients with } \\
\text { uncontrolled } \\
\text { acromegaly } \\
(n=31)\end{array}$} & \multicolumn{2}{|c|}{$\begin{array}{l}\text { Patients with } \\
\text { controlled } \\
\text { acromegaly } \\
(n=49)\end{array}$} & \multirow[t]{2}{*}{$\begin{array}{l}P \text {-value controlled } \\
\text { versus uncontrolled } \\
\text { acromegaly }\end{array}$} & \multicolumn{2}{|c|}{$\begin{array}{l}\text { DETECT-control } \\
\text { group, patients } \\
\text { with uncontrolled } \\
\text { acromegaly } \\
(n=120)\end{array}$} & \multirow[t]{2}{*}{$\begin{array}{l}P \text {-value versus } \\
\text { uncontrolled } \\
\text { acromegaly }\end{array}$} & \multicolumn{2}{|c|}{$\begin{array}{l}\text { DETECT- } \\
\text { control group, } \\
\text { patients with } \\
\text { controlled } \\
\text { acromegaly } \\
(n=196)\end{array}$} & \multicolumn{2}{|c|}{$\begin{array}{l}P \text {-value versus } \\
\text { controlled } \\
\text { acromegaly }\end{array}$} \\
\hline & $n$ & $\%$ & $n$ & $\%$ & & $n$ & $\%$ & & $n$ & $\%$ & & \\
\hline \multicolumn{13}{|l|}{ Comorbidities } \\
\hline Hypertension & 16 & 51.6 & 27 & 55.1 & 0.762 & 46 & 38.3 & 0.191 & 75 & 38.3 & 0.037 & \\
\hline $\begin{array}{l}\text { Diabetes } \\
\text { mellitus }\end{array}$ & 7 & 22.6 & 15 & 30.6 & 0.438 & 23 & 19.2 & 0.676 & 35 & 17.9 & 0.049 & \\
\hline $\begin{array}{l}\text { Coronary } \\
\text { heart disease }\end{array}$ & 4 & 12.9 & 3 & 6.1 & 0.309 & 11 & 9.2 & 0.544 & 19 & 9.7 & 0.443 & \\
\hline $\begin{array}{l}\text { Myocardial } \\
\text { infarction }\end{array}$ & 0 & 0.0 & 1 & 2.0 & - & 4 & 36.4 & - & 7 & 36.8 & 0.001 & \\
\hline $\begin{array}{l}\text { Cerebral } \\
\text { insult }\end{array}$ & 0 & 0.0 & 4 & 8.2 & - & 0 & 0.0 & - & 0 & 0.0 & - & \\
\hline \multirow[t]{2}{*}{ Malignancies } & 5 & 16.1 & 4 & 8.2 & 0.283 & 5 & 4.2 & 0.029 & 5 & 2.6 & 0.080 & \\
\hline & & & \multicolumn{2}{|r|}{ Mean (SD) } & Mean (SD) & \multicolumn{3}{|c|}{ Mean (SD) } & \multicolumn{4}{|c|}{ Mean (SD) } \\
\hline \multicolumn{13}{|c|}{ Anthropometric parameters } \\
\hline \multicolumn{3}{|l|}{ Height (cm) } & \multicolumn{2}{|r|}{$174.5(13.5)$} & $170.7(10.6)$ & 0.188 & \multicolumn{2}{|c|}{$170.9(9.9)$} & 0.165 & \multicolumn{2}{|c|}{$169.3(8.7)$} & 0.391 \\
\hline \multicolumn{3}{|l|}{ Weight (kg) } & \multicolumn{2}{|r|}{$87.1(17.2)$} & $85.6(17.9)$ & 0.707 & \multicolumn{2}{|c|}{$81.4(15.4)$} & 0.101 & \multicolumn{2}{|c|}{$78.5(18.5)$} & 0.015 \\
\hline \multicolumn{3}{|l|}{ BMI $\left(\mathrm{kg} / \mathrm{m}^{2}\right)$} & \multicolumn{2}{|r|}{$28.5(4.0)$} & $29.5(5.9)$ & 0.376 & \multicolumn{2}{|c|}{$27.9(5.2)$} & 0.523 & \multicolumn{2}{|c|}{$27.3(5.8)$} & 0.020 \\
\hline \multicolumn{3}{|c|}{ Waist circumference $(\mathrm{cm})$} & & $98.2(9.8)$ & $100.9(16.8)$ & 0.384 & & $.0(15.6)$ & 0.606 & 94.8 & 5.5) & 0.031 \\
\hline Hip circumfer & nce & & & $109.9(7.4)$ & $110.7(9.9)$ & 0.719 & 106 & $.5(12.6)$ & 0.063 & 105.0 & 1.7) & 0.001 \\
\hline Waist to heigh & t rati & & & $0.56(0.06)$ & $0.59(0.10)$ & 0.190 & & $57(0.09)$ & 0.712 & 0.56 & .09) & 0.089 \\
\hline Biochemical par & amet & & & & & & & & & & & \\
\hline IGF-1 ( $\mu \mathrm{g} / \mathrm{L})$ & & & & $317.1(216.7)$ & $149.1(47.7)$ & 0.000 & 135 & $.5(52.5)$ & 0.000 & 128.7 & 5.5) & 0.011 \\
\hline Cardiovascular 1 & isk b & narkers & & & & & & & & & & \\
\hline Fasting plasma & gluc & $(\mathrm{mg} / \mathrm{dL})$ & & $97.5(15.9)$ & 90.9 (16.7) & 0.084 & 103 & $.7(27.3)$ & 0.107 & 104.8 & 9.3) & 0.000 \\
\hline HbA1c (\%) & & & & $5.8(0.5)$ & $5.6(0.4)$ & 0.104 & & $6(0.9)$ & 0.069 & 5.7 & & 0.566 \\
\hline Total cholester & ol $(\mathrm{n}$ & dL) & & $204.6(42.1)$ & $223.6(36.8)$ & 0.058 & 225 & $.0(38.3)$ & 0.022 & 227.7 & 7.6) & 0.534 \\
\hline Triglycerides & $\mathrm{mg} / \mathrm{d}$ & & & $118.2(62.3)$ & $118.5(59.4)$ & 0.987 & 174 & $.4(198.5)$ & 0.011 & 153.8 & 23.1) & 0.006 \\
\hline HDL (mg/dL) & & & & $62.2(24.8)$ & $60.0(19.2)$ & 0.702 & & $.2(17.5)$ & 0.040 & 54.1 & 7.6) & 0.077 \\
\hline LDL (mg/dL) & & & & $128.8(40.1)$ & $146.9(34.2)$ & 0.070 & 129 & $6(28.5)$ & 0.929 & 131.9 & 4.8) & 0.013 \\
\hline Lipoprotein (a & $(\mathrm{mg}$ & & & $40.3(48.6)$ & $34.1(41.7)$ & 0.706 & & $.3(43.4)$ & 0.585 & 34.0 & 9.9) & 0.985 \\
\hline
\end{tabular}

Note: Significant effects are bold typed

No significant differences in anthropometric parameters remained between uncontrolled acromegalic patients and their controls after stratification, indicating that the observed differences between the acromegalic and the control group can be fully attributed to the controlled acromegalic patient group (Table 2).

In order to exclude type 2 diabetes mellitus as a potential influence on our calculations, we performed further stratified analyses with acromegalic patients and primary care controls, with and without the diagnosis of type 2 diabetes mellitus.
Comparisons of patients with acromegaly with and without diabetes mellitus and the respective primary care controls

Twenty-two acromegalic subjects were diagnosed with diabetes mellitus (age- and gender-matched control group of patients with diabetes mellitus $n=88$ ) during the course of the disease or treatment; 12 patients with acromegaly and diabetes mellitus had been treated with dopamine agonists, 12 with the somatostatin analogue octreotide, 5 with lanreotide and 4 with the GH receptor 
Table 3 Comparisons of comorbidities, anthropometric parameters and cardiovascular risk biomarkers between acromegalic patients with $(n=22)$ and without diabetes mellitus $(n=59)$ and the age- and gender-matched DETECT-controls $(n=88$ and $n=232$, respectively)

\begin{tabular}{|c|c|c|c|c|c|c|c|c|c|}
\hline & \multicolumn{2}{|c|}{$\begin{array}{l}\text { Patients with } \\
\text { acromegaly } \\
\text { and no diabetes } \\
\text { mellitus }+(n=59)\end{array}$} & \multicolumn{2}{|c|}{$\begin{array}{l}\text { Patients with } \\
\text { acromegaly and } \\
\text { diabetes mellitus } \\
(n=22)\end{array}$} & \multirow[t]{2}{*}{$\begin{array}{l}P \text {-value no } \\
\text { diabetes } \\
\text { versus } \\
\text { diabetes }\end{array}$} & $\begin{array}{l}\text { DETECT-control } \\
\text { group, patients } \\
\text { with no diabetes } \\
(n=232)\end{array}$ & \multirow[t]{2}{*}{$\begin{array}{l}\text { P-value } \\
\text { versus no } \\
\text { diabetes }\end{array}$} & $\begin{array}{l}\text { DETECT-control } \\
\text { group, patients } \\
\text { with diabetes } \\
(n=88)\end{array}$ & \multirow[t]{2}{*}{$\begin{array}{l}P \text {-value } \\
\text { versus } \\
\text { diabetes }\end{array}$} \\
\hline & $n$ & $\%$ & $n$ & $\%$ & & $\%$ & & $\%$ & \\
\hline \multicolumn{10}{|l|}{ Comorbidities } \\
\hline Hypertension & 29 & 49.2 & 15 & 68.2 & 0.133 & 37.9 & 0.007 & 37.5 & 0.715 \\
\hline Diabetes mellitus & - & - & - & - & & 18.1 & - & 18.2 & - \\
\hline Coronary heart disease & 5 & 8.5 & 2 & 9.1 & 0.930 & 10.3 & 0.437 & 6.8 & 0.130 \\
\hline Myocardial infarction & 1 & 1.7 & 0 & 0.0 & - & 29.2 & 0.002 & 66.7 & - \\
\hline Cerebral insult & 1 & 1.7 & 3 & 13.6 & 0.063 & 0.0 & - & 0.0 & - \\
\hline Malignancies & 6 & 10.2 & 3 & 13.6 & 0.662 & 3.0 & 0.015 & 3.4 & 0.227 \\
\hline & & \multicolumn{2}{|c|}{ Mean (SD) } & \multicolumn{2}{|l|}{ Mean (SD) } & Mean (SD) & \multicolumn{3}{|c|}{ Mean (SD) } \\
\hline \multicolumn{10}{|l|}{ Anthropometric parameters } \\
\hline Height $(\mathrm{cm})$ & & \multicolumn{2}{|c|}{$174.7(12.1)$} & $166.0(9.1)$ & 0.001 & $171.1(9.2)$ & 0.006 & $167.3(9.0)$ & 0.060 \\
\hline Weight $(\mathrm{kg})$ & & \multicolumn{2}{|c|}{$87.3(17.7)$} & $84.6(18.6)$ & 0.559 & $80.5(17.1)$ & 0.000 & $77.7(18.2)$ & 0.449 \\
\hline BMI $\left(\mathrm{kg} / \mathrm{m}^{2}\right)$ & & \multicolumn{2}{|c|}{$28.6(4.8)$} & $30.7(6.2)$ & 0.155 & $27.5(5.3)$ & 0.015 & $27.6(6.1)$ & 0.831 \\
\hline Waist circumference (c & & \multicolumn{2}{|c|}{$98.9(10.9)$} & $102.8(21.4)$ & 0.449 & $96.3(15.9)$ & 0.003 & $93.8(14.3)$ & 0.596 \\
\hline Hip circumference $(\mathrm{cm}$ & & \multicolumn{2}{|c|}{$110.8(8.7)$} & $110.2(10.7)$ & 0.830 & $105.6(12.1)$ & 0.000 & $105.6(11.9)$ & 0.829 \\
\hline Waist to height ratio & & \multicolumn{2}{|c|}{$0.57(0.06)$} & $0.61(0.13)$ & 0.131 & $0.56(0.09)$ & 0.127 & $0.56(0.08)$ & 0.870 \\
\hline \multicolumn{10}{|l|}{ Biochemical parameters } \\
\hline IGF-1 $(\mu \mathrm{g} / \mathrm{L})$ & & \multicolumn{2}{|c|}{$218.6(152.6)$} & $202.7(185.5)$ & 0.720 & $135.4(56.2)$ & 0.000 & $121.4(48.0)$ & 0.057 \\
\hline \multicolumn{10}{|c|}{ Cardiovascular risk biomarkers } \\
\hline \multicolumn{2}{|c|}{ Fasting plasma glucose $(\mathrm{mg} / \mathrm{dL})$} & \multicolumn{2}{|c|}{$89.9(11.9)$} & $103.6(22.8)$ & 0.010 & $104.3(36.8)$ & 0.011 & $103.5(30.2)$ & 0.000 \\
\hline & & \multicolumn{2}{|c|}{$5.6(0.4)$} & $5.9(0.6)$ & 0.038 & $5.6(1.0)$ & 0.000 & $5.7(0.9)$ & 0.000 \\
\hline \multicolumn{2}{|c|}{ Total cholesterol (mg/dL) } & 214.3 & & $219.8(41.7)$ & 0.616 & $227.1(45.9)$ & $\mathbf{0 . 0 3 1}$ & $225.3(38.9)$ & 0.787 \\
\hline Triglycerides (mg/dL) & & 107.1( & & $157.3(72.5)$ & 0.007 & $158.8(128.2)$ & 0.000 & $166.2(211.8)$ & 0.071 \\
\hline HDL (mg/dL) & & 59.9 & & $61.7(20.8)$ & 0.748 & $52.9(18.0)$ & 0.095 & $53.3(16.5)$ & 0.010 \\
\hline LDL (mg/dL) & & 139.7( & & $138.7(39.4)$ & 0.920 & $131.4(33.5)$ & 0.195 & $130.2(29.4)$ & 0.209 \\
\hline Lipoprotein (a) (mg/dL & & 45.6( & & $13.8(8.5)$ & 0.003 & $31.6(44.3)$ & 0.282 & $37.6(54.7)$ & 0.021 \\
\hline
\end{tabular}

Note: Significant effects are bold typed

antagonist pegvisomant (more than one therapy modality possible).

As presented in Table 3, we did not see significant differences in anthropometric parameters between acromegalic patients with diabetes mellitus and controls with diabetes mellitus. Additionally, the acromegalic patient group with diabetes mellitus even displayed an improved cardiovascular risk profile regarding HDL (61.7 \pm 20.8 vs. $53.3 \pm 16.5 \mathrm{mg} / \mathrm{dL} ; P=0.010)$ and lipoprotein (a) levels $(13.8 \pm 8.5$ vs. $37.6 \pm 54.7 \mathrm{mg} / \mathrm{dL} ; P=0.021)$, but not regarding total cholesterol, triglycerides and LDL. Acromegalic patients without diabetes mellitus presented significantly more often with hypertension (49.2 vs. $37.9 \%$; $P=0.007)$, but with lower fasting plasma glucose $(89.9 \pm 11.9$ vs. $104.3 \pm 36.8 \mathrm{mg} / \mathrm{dL} ; P=0.011)$, total cholesterol $(214.3 \pm 39.0$ vs. $227.1 \pm 45.9 \mathrm{mg} / \mathrm{dL}$;
$P=0.031)$ and triglycerides levels $(107.1 \pm 54.4 v s$. $158.8 \pm 128.2 \mathrm{mg} / \mathrm{dL} ; \quad P=0.000)$ compared to their respective controls.

\section{Discussion}

This study demonstrates that biochemically cured acromegalic patients exhibit an adverse anthropometric risk profile compared to an age- and gender-matched primary care control cohort.

Various factors could explain our reported findings. One possible reason for the adverse anthropometric measurements might be a relative "overtreatment of acromegaly" resulting in subsequent symptoms of relative growth hormone deficiency. However, in our study only three 
biochemically cured acromegalic patients had a low IGF-1 below two standard deviations as a potential marker of relative growth hormone deficiency, which is not sufficient to fully account for the observed effect.

Other pituitary deficiencies also might explain our findings, as suggested by Schmid et al. The authors found an increased prevalence of high BMI in patients with pituitary tumours, possibly caused by partial pituitary failure [17]. In our patient sample, 48 patients $(59.3 \%)$ suffered from pituitary deficiencies in total, and 19 patients $(23.5 \%)$ received hormonal replacement therapy with hydrocortisone. Inadequate dosages or overtreatment could have caused the adverse anthropometric profile in these patients.

Another factor could be that acromegalic patients lack physical exercise because of persisting joint complaints or arthropathy, as reported by Biermasz et al. [18]. As arthralgia was reported by $65.4 \%$ of the patients in this cohort, this might have contributed greatly to the observed effect [19].

Additionally, the medical treatment itself might lead to the increased measurements for body mass and body fat. Plöckinger et al. [20], for instance, proposed a significant increase in intra-abdominal fat mass during therapy with the GH receptor antagonist pegvisomant, while subcutaneous fat was not significantly different. In this acromegalic cohort, the significant number of nine patients $(11 \%)$ were being treated with pegvisomant at the time of study which might explain a large proportion of the observed results.

As a limitation of our study, we have to consider that the measurements reported here are restricted to anthropometric parameters without direct measurements of lean tissue mass or fat mass, since a DEXA measurement or other more site-specific measurements were not part of the DETECT study. On the other hand, anthropometric measurements alone are already strong indicators for morbidity and mortality, as published by Schneider et al. [21]. We therefore believe that they should be sufficiently valid.

We can only speculate as to why some cardiovascular risk biomarkers, such as triglycerides, are more favourable than in the control group. One possible explanation could be that acromegalic patients are more aggressively treated than the control group with, i.e., a lipid lowering therapy, because of the known cardiovascular risk profile. However, we did not analyse this systematically.

Furthermore, some measurements in acromegalic patients versus DETECT subjects had to be performed with different laboratory methods, possibly yielding systematic differences and influencing our findings. However, both laboratories are part of routine standardisation procedures and should therefore deliver comparable results. Additionally, most of the examined biochemical parameters were measured with the same assays.

Moreover, cardiovascular risk biomarkers are only markers and the study was not designed to analyse "hard" end points, such as incidence of hypertension, myocardial infarction or stroke.

We also have to consider that we are comparing to a primary care patient population which is not representative for the general population, since primary care patients can be expected to be less healthy than the general population.

Nevertheless, we believe that the selection of the control group represents a valid comparison because it contrasts to a group that seeks medical advice for other reasons, reflecting a realistic scenario.

How the reported findings might influence the current management paradigm of acromegaly is not clear. However, stricter treatment goals of cardiovascular risk factors targeting mainly overweight and visceral adiposity in these patients are likely to be beneficial.

Acknowledgments We thank Iris Miedl, Doris Goy-Alsing and Sylvia Lang for their valuable contribution in helping to process the patient data. Diabetes cardiovascular risk-evaluation: targets and essential data for commitment of treatment (DETECT) is a crosssectional and prospective-longitudinal, nationwide clinical epidemiological study. DETECT is supported by an unrestricted educational grant from Pfizer GmbH, Karlsruhe, Germany. Principal investigator: Prof. Dr. H. -U. Wittchen; Staff members: Dr. H. Glaesmer, E. Katze, Dipl.-Math. J. Klotsche, Dipl.-Psych. L. Pieper, Dipl.-Psych. A. Bayer, Dipl.-Psych. A. Neumann. Steering Committee: Prof. Dr. H. Lehnert (Magdeburg, Coventry), Prof. Dr. G. K. Stalla (Munich), Prof. Dr. M. A. Zeiher (Frankfurt), Professor Dr. M. Wehling (Mannheim); Advisory Board: Prof. Dr. W. März (Graz/Heidelberg), Prof. Dr. S. Silber (Munich), Prof. Dr. Dr. U. Koch (Hamburg), PD Dr. D. Pittrow (Munich, Dresden).

Declaration of interest None of the authors report a conflict of interest.

Financial disclosure This study was funded by Pfizer Pharma GmbH, Karlsruhe, Germany. Two co-authors (C. S. and G. K. S.) were supported by a research grant from Pfizer Pharma GmbH, Karlsruhe, Germany. G. K. S. received lecture fees from Pfizer Pharma GmbH, Karlsruhe, Germany, Novo Nordisk Pharma GmbH, Germany, Ipsen International GmbH, Germany, and Novartis Pharma GmbH, Germany. H. J. S. received lecture fees from Novo Nordisk Pharma GmbH, and Pfizer Pharma GmbH, Karlsruhe, Germany and is a member of the German KIMS Board (Pfizer). C. S. received lecture fees from Pfizer Pharma GmbH, Karlsruhe, Germany, and Novartis Pharma GmbH, Germany.

Open Access This article is distributed under the terms of the Creative Commons Attribution Noncommercial License which permits any noncommercial use, distribution, and reproduction in any medium, provided the original author(s) and source are credited.

\section{References}

1. Daly AF, Rixhon M, Adam C, Dempegioti A, Tichomirowa MA, Beckers A (2006) High prevalence of pituitary adenomas: a cross-sectional study in the province of Liege, Belgium. J Clin Endocrinol Metab 91:4769-4775 
2. Holdaway IM, Rajasoorya C (1999) Epidemiology of acromegaly. Pituitary 2:29-41

3. Colao A, Ferone D, Marzullo P, Lombardi G (2004) Systemic complications of acromegaly: epidemiology, pathogenesis, and management. Endocr Rev 25:102-152

4. Fedrizzi D, Czepielewski MA (2008) Cardiovascular disturbances in acromegaly. Arq Bras Endocrinol Metabol 52:1416-1429

5. Colao A (2008) The GH-IGF-I axis and the cardiovascular system: clinical implications. Clin Endocrinol (Oxf) 69:347-358

6. Feelders RA, Hofland LJ, van Aken MO, Neggers SJ, Lamberts SW, de Herder WW, van der Lely AJ (2009) Medical therapy of acromegaly: efficacy and safety of somatostatin analogues. Drugs 69:2207-2226

7. Melmed S (2009) Acromegaly pathogenesis and treatment. J Clin Invest 119:3189-3202

8. Freda PU, Shen W, Heymsfield SB, Reyes-Vidal CM, Geer EB, Bruce JN, Gallagher D (2008) Lower visceral and subcutaneous but higher intermuscular adipose tissue depots in patients with growth hormone and insulin-like growth factor I excess due to acromegaly. J Clin Endocrinol Metab 93:2334-2343

9. O'Sullivan AJ, Kelly JJ, Hoffman DM, Freund J, Ho KK (1994) Body composition and energy expenditure in acromegaly. J Clin Endocrinol Metab 78:381-386

10. Tominaga A, Arita K, Kurisu K, Uozumi T, Migita K, Eguchi K, II K, Kawamoto H, Mizoue T (1998) Effects of successful adenomectomy on body composition in acromegaly. Endocr $\mathrm{J}$ 45:335-342

11. Sucunza N, Barahona MJ, Resmini E, Fernandez-Real JM, Farrerons J, Lluch P, Puig T, Wagner AM, Ricart W, Webb SM (2008) Gender dimorphism in body composition abnormalities in acromegaly: males are more affected than females. Eur J Endocrinol 159:773-779

12. Boero L, Manavela M, Gomez Rosso L, Insua C, Berardi V, Fornari M, Brites F (2009) Alterations in biomarkers of cardiovascular disease in active acromegaly. Clin Endocrinol (Oxf) 70:88-95
13. Potter BJ, Beauregard C, Serri O (2008) Serum markers of cardiovascular risk in patients with acromegaly before and after 6 months of treatment with octreotide LAR. Pituitary 11:49-53

14. Wittchen HU, Glaesmer H, Marz W, Stalla G, Lehnert H, Zeiher AM, Silber S, Koch U, Bohler S, Pittrow D et al (2005) Cardiovascular risk factors in primary care: methods and baseline prevalence rates-the DETECT program. Curr Med Res Opin 21:619-630

15. Pieper L, Wittchen HU, Glaesmer H, Klotsche J, Marz W, Stalla G, Lehnert H, Zeiher AM, Silber S, Koch U et al (2005) Cardiovascular high-risk constellations in primary care. DETECT Study 2003. Bundesgesundheitsblatt Gesundheitsforschung Gesundheitsschutz 48:1374-1382

16. Elmlinger MW, Kuhnel W, Weber MM, Ranke MB (2004) Reference ranges for two automated chemiluminescent assays for serum insulin-like growth factor I (IGF-I) and IGF-binding protein 3 (IGFBP-3). Clin Chem Lab Med 42:654-664

17. Schmid C, Goede DL, Hauser RS, Brandle M (2006) Increased prevalence of high body mass index in patients presenting with pituitary tumours: severe obesity in patients with macroprolactinoma. Swiss Med Wkly 136:254-258

18. Biermasz NR, Pereira AM, Smit JW, Romijn JA, Roelfsema F (2005) Morbidity after long-term remission for acromegaly: persisting joint-related complaints cause reduced quality of life. J Clin Endocrinol Metab 90:2731-2739

19. Sievers C, Dimopoulou C, Pfister H, Lieb R, Steffin B, Roemmler J, Schopohl J, Mueller M, Schneider HJ, Ising M et al (2009) Prevalence of DSMIV mental disorders in acromegaly: a crosssectional study in 81 acromegalic patients. Clin Endocrinol (Oxf) 71:691-701

20. Plockinger U, Reuter $\mathrm{T}$ (2008) Pegvisomant increases intraabdominal fat in patients with acromegaly: a pilot study. Eur $\mathrm{J}$ Endocrinol 158:467-471

21. Schneider HJ, Klotsche J, Stalla GK, Wittchen HU (2006) Obesity and risk of myocardial infarction: the INTERHEART study. Lancet 367:1052 Author reply 1054 